\title{
Effect of Pulsed High-Voltage Stimulation on Pholiota Nameko Mushroom Yield
}

\author{
K. TAKaki ${ }^{a * *}$, N. YAmazaki ${ }^{a}$, S. Mukaigawa ${ }^{a}$, T. Fujiwara ${ }^{a}$, H. Kofujita $^{a}$, \\ K. TAKAHASI ${ }^{b}$, M. NARIMATSU $^{c}$ AND K. NAGANE ${ }^{d}$ \\ ${ }^{a}$ Iwate University, Ueda 4-3-5, Morioka, Iwate 020-8551, Japan \\ ${ }^{b}$ Morioka Forest Association, Konya-cho 2-9, Morioka, Iwate 020-0885, Japan \\ ${ }^{c}$ Tono Agricultural and Forestry Center, Muikamachi 1-22, Tono, Iwate 028-0525, Japan \\ ${ }^{d}$ Nagane Co. Ltd., Nakano 3-37-41, Hirono-cho, Kunohe-gun, Iwate 028-0790, Japan
}

\begin{abstract}
A pulsed high voltage was applied to logs for mushroom cultivation to investigate an effect of pulse high voltage on mushroom growth. Inductive energy storage system was utilized to construct a pulsed power generator with compact size. Copper fuse of $0.03 \mathrm{~mm}$ diameter was used as an opening switch to interrupt large circuit current in short time. The voltage charged in primary energy storage capacitor was multiplied using secondary energy storage inductor. The output voltage of the pulsed power generator was $120 \mathrm{kV}$ with $100 \mathrm{~ns}$ pulse width at $14 \mathrm{kV}$ charging voltage of $0.22 \mu \mathrm{F}$ primary energy storage capacitor and $15 \mu \mathrm{H}$ secondary energy storage inductor. This pulsed high voltage was applied to $90 \mathrm{~cm}$ length logs inoculated Pholiota nameko fungus. The yield of Pholiota nameko mushrooms was successfully improved with the pulse voltage stimulation. The total weight of the cropped Pholiota nameko after the pulse voltage stimulation was $6.3 \mathrm{~kg}$ for fifteen logs and this value was 1.5 times larger than $4.3 \mathrm{~kg}$ of total weight without the stimulation. The number of logs with lower than $0.27 \mathrm{~kg}$ cropped Pholiota nameko weight which was an average value for control group was only one in fifteen, whereas four logs in control group showed almost zero weight of cropped Pholiota nameko.
\end{abstract}

PACS numbers: 87.50.-a, 52.90.+z, 82.39.Rt

\section{Introduction}

Mushrooms extraordinary grow-up around a hit point of a lightning have been reported by some mushroom farmers [1]. The mechanism of the mushroom outbreak is not clear, but some researchers suggest two possibilities. One is the generation of cracks in mycelium hyphae by the lightning because mushroom fruiting bodies are generated from cracks in hyphae [1]. The other is the activity of enzymes. Some enzymes were activated by applying pulse electric field and, as the results, mushroom fruiting bodies were actively developed [2].

An effectiveness of electrical sources such as high voltages in sinusoidal waves or pulses in cultivating mushrooms has been reported by several researchers since the 1950s. In 1984, Mibuchi et al. reported that the yield of shiitake mushrooms (Lentinula edodes) increased by electrical stimulation [3]. They achieved the improvement of mushroom yield to be double by applying an impulse high voltage with $40 \mu$ s pulse width. Some other researchers also achieved the improvement of mushroom yield using other electrical sources such as high voltages

\footnotetext{
* corresponding author; e-mail: takaki@iwate-u.ac.jp
}

of sinusoidal waves or short width pulses [2-5]. For the commercial use of the cultivating technique, it is necessary to develop compact and portable high-voltage power sources.

Inductive energy storage (IES) pulsed power generators have favourable features for the mushroom culture applications, e.g. compact size, cost effectiveness, light weight, high voltage amplification compared with capacitive energy storage generators such as the impulse generator [6]. Tsukamoto et al. carried out high voltage pulse stimulation in Lentinula edodes cultures using IES pulsed power generator [7]. However, it is not clear that an effect of the high-voltage stimulation for other kinds of mushrooms such as a nameko mushroom (Pholiota nameko) which is mainly cultured at north part of Japan; Tohoku area. In this paper, the effect of high-voltage pulse stimulation applied with IES pulsed power generator on Pholiota nameko mushroom yield is described.

\section{Behaviour of pulsed power generator}

Figure 1 shows the IES pulsed power generator circuit used for the experiment of pulse voltage stimulation of the nameko mushrooms. The IES pulsed power generator basically consists of a primary energy storage capacitor 
$C$, a closing switch $G S$, a secondary energy storage inductor $L$ and an opening switch [8]. Copper fuse of $0.03 \mathrm{~mm}$ diameter was used as the opening switch to interrupt large current in short time. The primary energy storage capacitor of $0.22 \mu \mathrm{F}$ was charged up using high voltage dc power supply (50 kV maximum voltage). A charging voltage $V_{0}$ of the primary energy storage capacitor $C$ and an inductance of the secondary energy storage inductor $L$ were changed in range from 10 to $16 \mathrm{kV}$ and from 1.3 to $38 \mu \mathrm{H}$, respectively. The circuit current and the output voltage were measured with Pearson 110A current transformer and Pulse Electronics EP-100K high-voltage probe, respectively. The output signals from the current transformer and the voltage probe were monitored using a Tektronix TDS3054B digitizing oscilloscope.

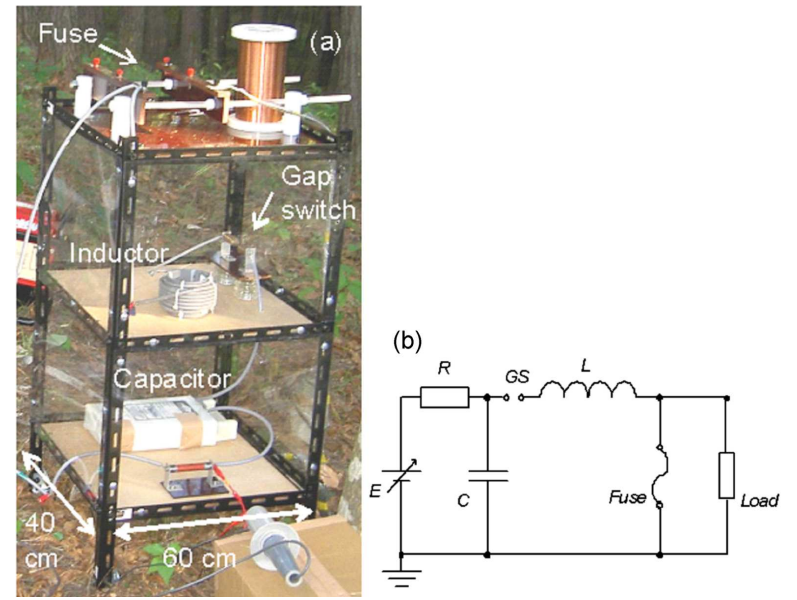

Fig. 1. IES pulsed power generator photograph (a) and its circuit with fuse opening switch (b) $(C$ : Primary energy storage capacitor, $L$ : Secondary energy storage inductor).

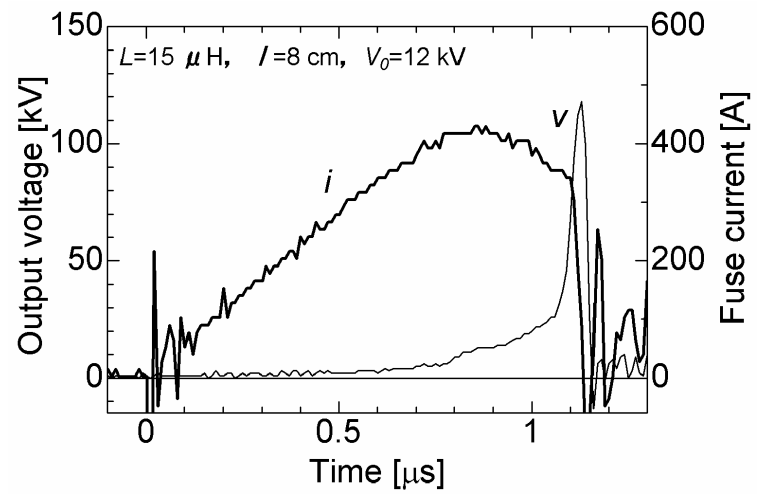

Fig. 2. Typical waveforms of circuit current through the fuse and output voltage at $12 \mathrm{kV}$ charging voltage, $8 \mathrm{~cm}$ fuse length and $15 \mu \mathrm{H}$ inductance.

Figure 2 shows typical circuit (fuse) current and output voltage waveforms without connection to the logs at $12 \mathrm{kV}$ charging voltage. The fuse length and the in- ductance of the secondary energy storage inductor are chosen to be $8 \mathrm{~cm}$ and $15 \mu \mathrm{H}$, respectively. The time 0 means closing the switch $G S$. The circuit current starts to flow after closing the switch $G S$ with $L C$ oscillation. The peak value of the circuit current is $430 \mathrm{~A}$ at around $0.85 \mu \mathrm{s}$ after closing the gap switch. The circuit current decreases gradually from 430 to 350 A with increasing time from 0.85 to $1.1 \mu \mathrm{s}$. This time duration corresponds to a fuse melting phase. The circuit current is interrupted after fuse melting phase within $50 \mathrm{~ns}$. The output voltage increases rapidly and has a maximum voltage of $120 \mathrm{kV}$, which means that an amplification factor defined as the ratio of the maximum output voltage to the charging voltage is equal to 10 . The pulse width of the output voltage is $50 \mathrm{~ns}$ in FWHM (full-width at half-maximum). The high-voltage pulse is produced by the total circuit inductance and rapid current interruption produces a high-voltage pulse expressed as

$$
V=V_{0}-\frac{1}{C} \int i \mathrm{~d} t-L \frac{\mathrm{d} i}{\mathrm{~d} t} \approx-L \frac{\mathrm{d} i}{\mathrm{~d} t},
$$

where $i$ means the circuit current.

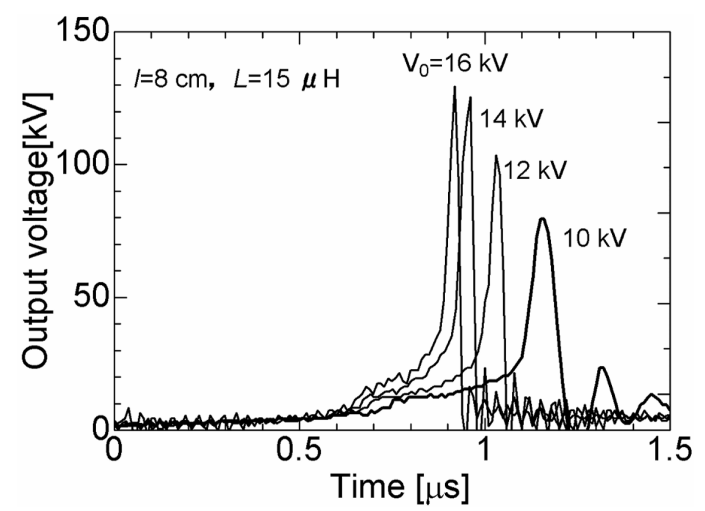

Fig. 3. Output voltage waveforms for various charging voltages at $8 \mathrm{~cm}$ fuse length and $15 \mu \mathrm{H}$ inductance.

Figure 3 shows the output voltage waveforms for various charging voltages with $8 \mathrm{~cm}$ fuse length and $15 \mu \mathrm{H}$ inductance. The time duration from closing the switch $G S(t=0)$ to peak of the output voltage decreases from 11.5 to $0.92 \mu$ s with increasing charging voltage from 10 to $16 \mathrm{kV}$. The time duration of the fuse melting phase also decreases from 0.35 to $0.20 \mu$ s with increasing charging voltage from 10 to $16 \mathrm{kV}$. The increase of energy deposited into the fuse by increasing the charging voltage causes the decrease of the time duration for fuse melting and, as the result, also causes the decrease of the time duration for current interruption. The amplitude of the voltage peak increases with increasing the charging voltage and are $79.5,103.5,125.5$ and $129.5 \mathrm{kV}$ for 10 , 12,14 and $16 \mathrm{kV}$ charging voltage, respectively. These values correspond to values of $8.0,8.6,9.0$ and 8.1 of voltage amplification factors. The result indicates that the voltage amplification factor increases with charging voltage and has a maximum value. The voltage amplifi- 


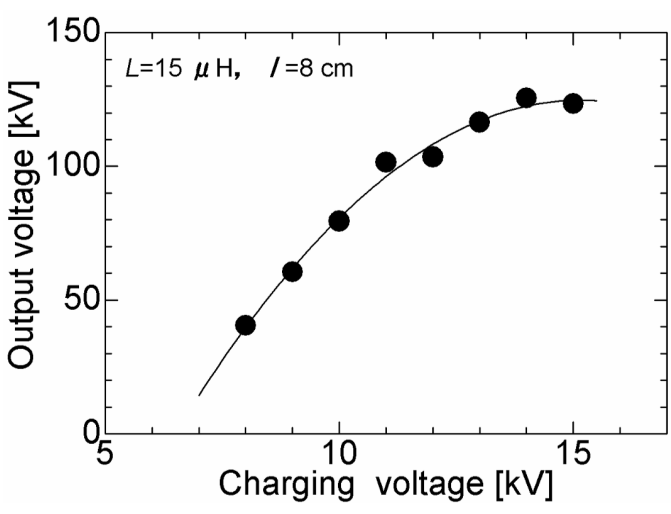

Fig. 4. Peak output voltage as a function of charging voltage at $8 \mathrm{~cm}$ fuse length and $15 \mu \mathrm{H}$ inductance.

cation factor decreases with charging voltage higher than $14 \mathrm{kV}$. Figure 4 shows the peak output voltage as a function of charging voltage at $8 \mathrm{~cm}$ fuse length and $15 \mu \mathrm{H}$ inductance. The peak output voltage increases with increasing the charging voltage and saturates around $14 \mathrm{kV}$ charging voltage. In general, the maximum output voltage is limited by breakdown voltage between the fuse [8]. Therefore, the electric field for breakdown is estimated to be $15.7 \mathrm{kV} / \mathrm{cm}$ for $125.5 \mathrm{kV}$ at $8 \mathrm{~cm}$ fuse length.

\section{Effect of pulsed power stimulation}

Figure 5 shows experimental setup for pulsed power stimulation applied to the Pholiota nameko logs. The Pholiota nameko logs have the length of $90 \mathrm{~cm}$ and the diameter of around $15 \mathrm{~cm}$. The logs were inoculated Pholiota nameko fungus on April of 2004. The strain of the Pholiota nameko hypha has fruiting seasons in autumn. After inoculation of Pholiota nameko fungus, they were

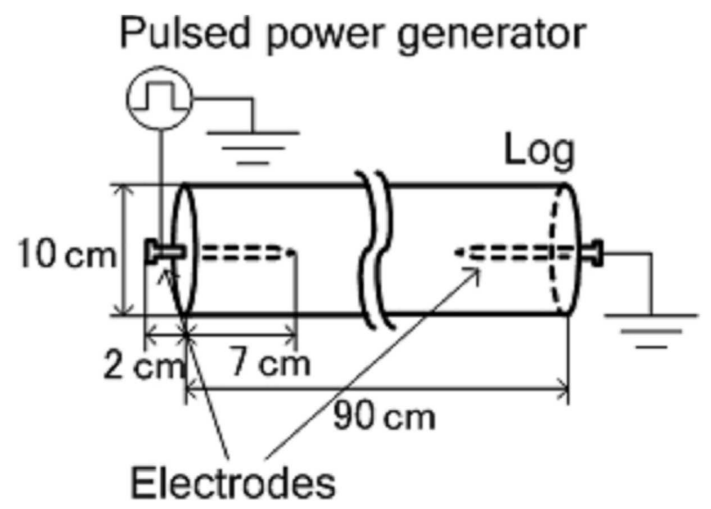

Fig. 5. Experimental setup for pulsed power stimulation to the Pholiota nameko logs.

matured in the woods during period necessary for each experiment. The experiment was carried out as an attempt to increase the yields of Pholiota nameko in autumn of 2006. We obtained the data of total weight of

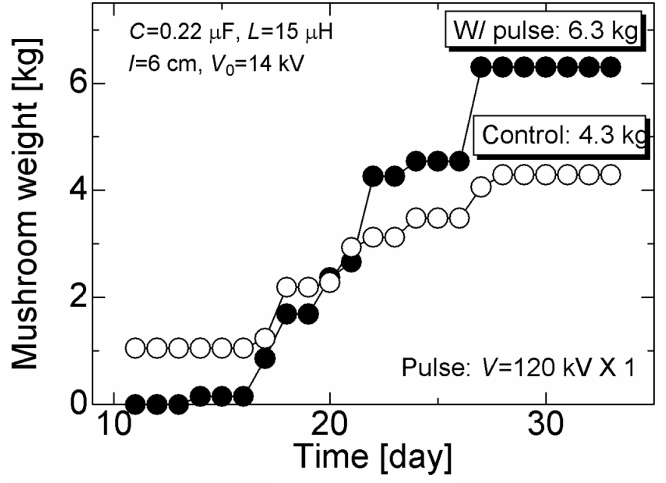

Fig. 6. Total weight of cropped nameko mushrooms as a function of days from pulsed power stimulation at $120 \mathrm{kV}$ applied voltage.

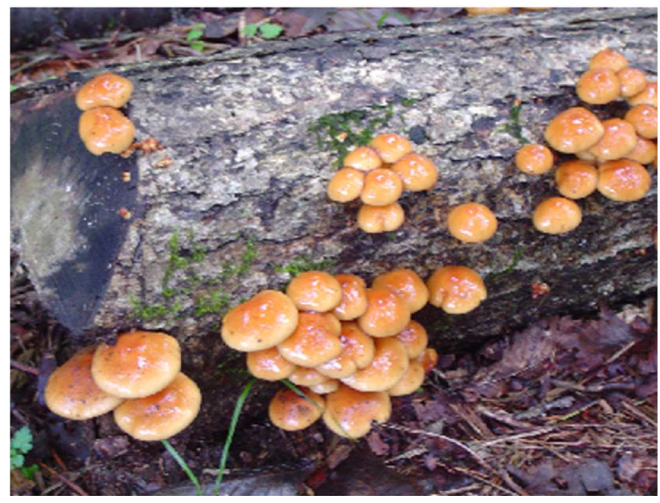

Fig. 7. Photograph of cultured Pholiota nameko.

Pholiota nameko using all flush (fruit body formation) for the log history. Logs of 15 were used as applying voltage group. All data were the total value of 15 logs. The logs of applying voltage group were stimulated with the pulsed voltage of $120 \mathrm{kV} ; 14 \mathrm{kV}$ charging voltage, $15 \mu \mathrm{H}$ inductance and $6 \mathrm{~cm}$ fuse length. Fifteen logs of the control group were not stimulated with the pulse voltage. Another fifteen logs were applied with the $120 \mathrm{kV}$ pulse voltage as the stimulation on 5th October of 2006.

Fruiting bodies of nameko mushrooms were cropped from 15 th October to 17 th November. The mushrooms were cropped every morning during the cropping term. Figure 6 shows the total weight of nameko mushroom cropped by 15 logs as a function of days from the pulsed power stimulation for the both groups. The $120 \mathrm{kV}$ pulse voltage was applied as the stimulation at once.

Figure 7 shows the photograph of cultured Pholiota nameko. The Pholiota nameko start to appear about two weeks after the stimulation and stop to appear at day of 26th. The yield of Pholiota nameko is successfully improved with the pulse voltage stimulation as shown in Fig. 6. The total weight of the cropped Pholiota nameko with the pulse voltage stimulation is $6.3 \mathrm{~kg}$ for fifteen $\operatorname{logs}$ and this value was 1.5 times larger than $4.3 \mathrm{~kg}$ of total weight without the stimulation. 


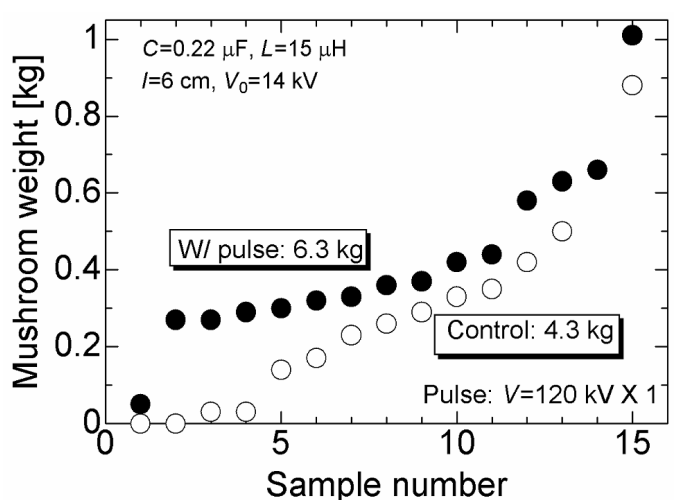

Fig. 8. Total weight of nameko mushrooms cropped by each $\log$ for stimulated and control groups at $120 \mathrm{kV}$ applied voltage.

Figure 8 shows the total weight of nameko mushroom cropped by each log for stimulated and control groups. The cropped mushroom weights from the logs with the stimulation are larger than those without the stimulation, whereas the data has some scattering. The number of logs with lower than $0.27 \mathrm{~kg}$ cropped Pholiota nameko weight which is an average value for control group is only one in fifteen, whereas four logs in control group shows almost zero weight of cropped Pholiota nameko.

\section{Conclusions}

The pulsed high voltage was applied to logs for mushroom cultures to investigate an effect of pulse high-voltage on the growth. The inductive energy storage system was employed to realize a compact pulsed power generator. The copper thin fuse was used as opening switch to interrupt large circuit current in short time. The fuse had the dimension of $0.03 \mathrm{~mm}$ diameter and $6 \mathrm{~cm}$ length. The output voltage of the pulsed power generator was $120 \mathrm{kV}$ with $100 \mathrm{~ns}$ pulse width. This $120 \mathrm{kV}$ high-voltage pulse was applied to $90 \mathrm{~cm}$ length logs inoculated nameko fungus in October of 2006. The fifteen logs were applied the pulsed voltage and were cultivated for a month. The results showed that the yield of Pholiota nameko mushrooms was successfully improved with the pulse voltage stimulation. The total weight of the cropped Pholiota nameko with the pulse voltage stimulation was $6.3 \mathrm{~kg}$ for fifteen logs and this value was 1.5 times larger than $4.3 \mathrm{~kg}$ of total weight without the stimulation. The number of logs lower than $0.27 \mathrm{~kg}$ cropped Pholiota nameko weight which was an average value for control group was only one in fifteen, whereas four logs in control group showed almost zero weight of cropped Pholiota nameko.

\section{Acknowledgments}

The author thanks Drs. S. Tsukamoto of Ariake National College of Technology, H. Akiyama of Kumamoto University, S. Kajiwara of Iwate University, T. Tateishi of Iwate University for their valuable comments and discussions. The authors thank Y. Shida, a staff member of Iwate University, T. Sasaki, a student of Iwate University for their technical supports. The work was supported by the Sanriku Kikin Foundation.

\section{References}

[1] S. Tsukamoto, H. Kudoh, S. Oga, K. Yamamoto, H. Akiyama, Proc. 15th Pulsed Power Conf., 1437 (2005).

[2] S. Ohga, S. Iida, J. Forest Res. 6, 37 (2001).

[3] Y. Mibuchi, M. Yamamoto, Kyushu Electric Power Co. Research Report No.87004, 1 (1984) [in Japanese].

[4] K. Kudo, K. Mitobe, N. Yoshimura, J. Institute Electrostatics Japan 23, 186 (1999) [in Japanese].

[5] S. Ohga, S. Iida, C.D. Koo, N.S. Cho, J. Japanese Soc. Mushroom Science Biotechnology 9, 7 (2001).

[6] K. Takaki, H. Kirihara, C. Noda, S. Mukaigawa, T. Fujiwara, Plasma Process Polymer 3, 734 (2006).

[7] S. Tsukamoto, T. Maeda, M. Ikeda, H. Akiyama, Proc. 14th Pulsed Power Conference, 1116 (2003).

[8] K. Takaki, K. Kanesawa, S. Mukaigawa, T. Fujiwara, T. Go, IEEE Trans. Diel. Elec. Insul. 14, 834 (2007). 